\title{
High Temperature Stability of Amorphous Zn-Sn-O Transparent Conductive Oxides Investigated by In Situ TEM and X-ray Diffraction
}

\author{
Q. Jeangros ${ }^{1}$, M. Duchamp ${ }^{2}$, E. Rucavado ${ }^{1}$, F. Landucci ${ }^{1,3}$, C. Spori ${ }^{1}$, R.E. Dunin-Borkowski ${ }^{2}$, \\ C. Hébert ${ }^{3}$, M. Morales-Masis ${ }^{1}$, C. Ballif ${ }^{1}$, A. Hessler-Wyser ${ }^{1}$ \\ 1. Photovoltaics and Thin Film Electronics Laboratory, Ecole Polytechnique Fédérale de Lausanne, \\ Neuchâtel, Switzerland \\ 2. Ernst Ruska-Centre for Microscopy and Spectroscopy with Electrons and Peter Grünberg Institute, \\ Jülich Research Centre, Jülich, Germany \\ 3. Interdisciplinary Centre for Electron Microscopy, Ecole Polytechnique Fédérale de Lausanne, \\ Lausanne, Switzerland
}

Transparent conductive oxides (TCOs) are used in a wide variety of technologies, including photovoltaic cells, organic light-emitting diodes, low-emissivity windows and flat-panel displays. While indiumbased compounds are the most widely employed TCOs due to their good optical and electrical properties, they are expensive as a result of the scarcity of indium. Therefore, there is strong interest in the development of alternative oxides that contain only earth-abundant elements. In addition to presenting equivalent electrical and optical properties to those of In-based TCOs, these alternative compounds should also exhibit good thermal stability for compatibility with device fabrication.

Based on these criteria, a compound of Zn-Sn-O (ZTO) is selected here and thin films of defect-free amorphous (a-) ZTO are deposited by sputtering [1]. Transmission electron microscopy (TEM) imaging, fluctuation electron microscopy (FEM [2]) and X-ray diffraction (XRD) experiments, all performed in situ, are then used to assess changes in the crystallography of a-ZTO upon annealing in different atmospheres. The results are correlated with optical and electrical properties.

The crystallization kinetics of a-ZTO is found to be strongly influenced by the annealing atmosphere and, when the specimen is heated while being irradiated by the electron beam in the TEM, by sample preparation. Samples deposited on standard aluminoborosilicate glass and prepared by conventional focused ion beam (FIB) milling and lift-out start to crystallize as $\mathrm{SnO}_{2}$ at room temperature as a result of an interaction between the electron beam, the glass support and the a-ZTO. The crystalline interfacial layer then influences crystallization dynamics observed at higher temperatures, with metallic Sn nanoparticles nucleating in the film (Fig. 1). In contrast, the crystallization kinetics of films deposited directly on Si-based heating chips (coated by a thin $\mathrm{SiN}$ layer) exhibit completely different behavior, with a-ZTO crystallizing directly into $\mathrm{SnO}_{2}$ at $\sim 900{ }^{\circ} \mathrm{C}$, consistent with in situ X-ray diffraction experiments performed under similar conditions. For this sample architecture, FEM was performed up to $900{ }^{\circ} \mathrm{C}$. The results reveal that the atomic structure of a-ZTO does not appear to reorganize into intermediate "ordered" clusters before crystallization, i.e., the transition from a-ZTO to c- $\mathrm{SnO}_{2}$ is sharp. When annealed in air, XRD shows that a-ZTO crystallizes into $\mathrm{SnO}_{2}$, but at a lower temperature $\left(\sim 550^{\circ} \mathrm{C}\right)$, indicating that the incorporation of gaseous oxygen into the film lowers the activation energy for crystallization. In both air and vacuum, $\mathrm{Zn}$ starts to evaporate from the film once it crystallizes due to the low solubility of $\mathrm{Zn}$ in $\mathrm{c}-\mathrm{SnO}_{2}$. This behavior is detrimental to the electrical properties of the TCO, as it leads to the formation of a defective microstructure. On a technological level, the high thermal stability of the defect-free amorphous ZTO microstructure in oxygen-poor atmospheres may enable its application in high efficiency photovoltaic architectures [3]. 


\section{References:}

[1] M Morales-Masis et al., Advanced Functional Materials 26 (2016), p. 384.

[2] PM Voyles, DA Muller, Ultramicroscopy 93 (2002), p. 147.

[3] F Feldmann et al., Applied Physics Letters 104 (2014), p. 181105.

[4] Support is gratefully acknowledged from the Swiss National Science Foundation (project CRSII2_154474, "Impact of composition and nanometer scale DISorder in transparent Conductive Oxides: a new route to design materials with enhanced transport properties (DisCO)"). The authors wish to thank Dr. O. Sereda, Dr. I. Marozau and J. Tillier for access to an in situ X-ray diffractometer.
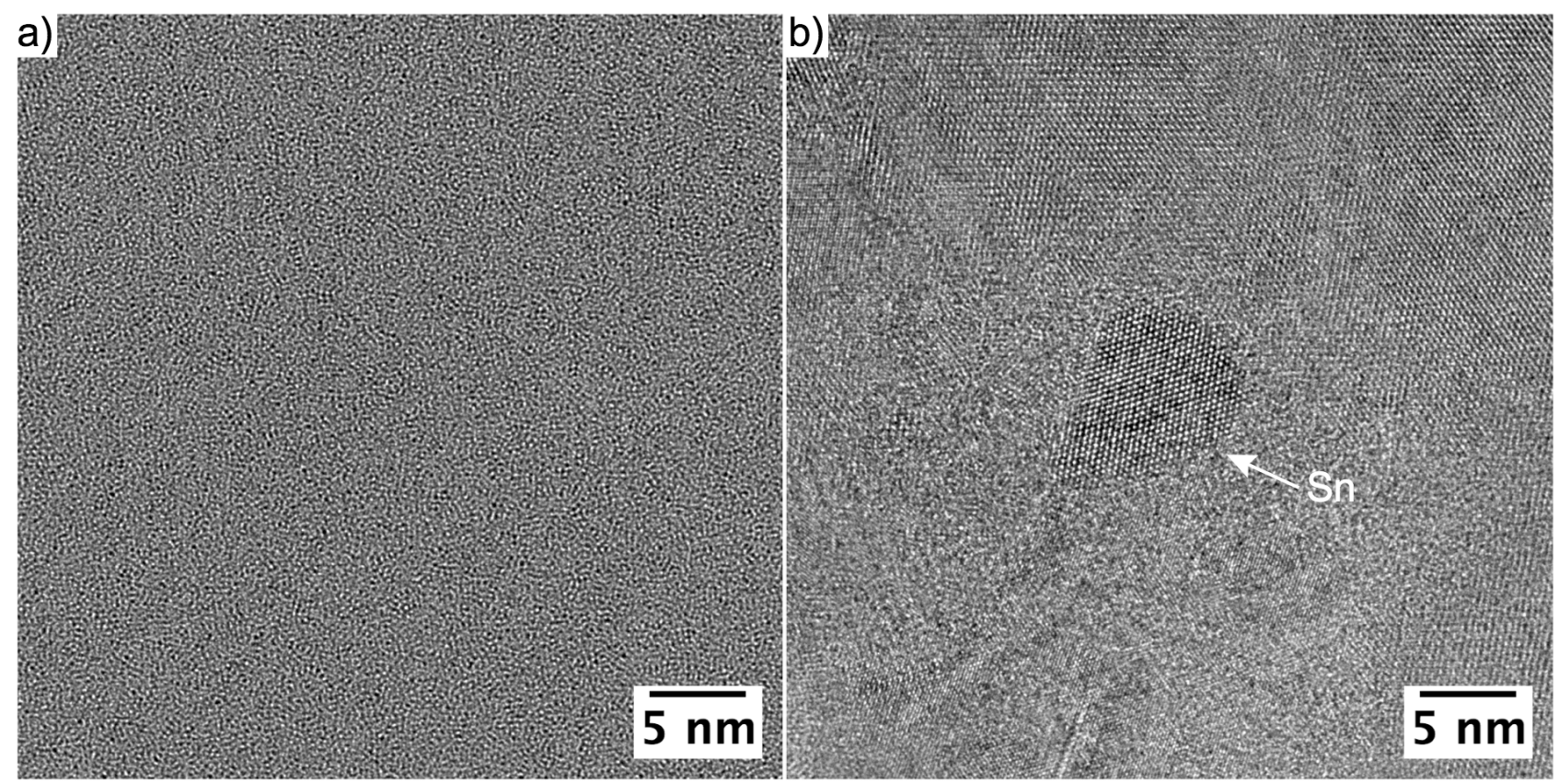

Figure 1. High-resolution TEM images of a) the initial a-ZTO film prepared by the conventional FIB lift-out technique and b) the film after annealing in situ in the microscope up to $500{ }^{\circ} \mathrm{C}$, highlighting the nucleation of Sn nanoparticles during the process. 